\title{
CONSUMO DE PSICOATIVOS LÍCITOS DURANTE A PANDEMIA DE COVID-19
}

\section{Letícia Galloni Menichelli', Lucas Rodrigueiro de Freitas', Rodrigo Vieira Gonzaga ${ }^{2, A}$}

${ }^{1}$ Discente do curso de Farmácia da Universidade Anhembi Morumbi - São Paulo, SP - Brasil

${ }^{2}$ Docente da Escola de Ciências da Saúde e Bem-Estar - Universidade Anhembi Morumbi - São Paulo, SP - Brasil

\section{RESUMO}

Objetivos: O objetivo deste estudo foi realizar uma análise comportamental da utilização de psicoativos lícitos durante a pandemia da Covid-19. Metodologia: Realizou-se uma busca bibliográfica de materiais disponíveis em meio eletrônicos de diversas bases de dados renomadas: Elsevier, Google Scholar, Literatura Latino Americana e do Caribe em Ciências da Saúde - LILACS, Biblioteca Nacional de Medicina do Instituto Nacional de Saúde dos Estados Unidos (PubMed Central - PMC) e SciELO. Foram selecionados 39 materiais, considerando o fator de impacto das publicações. Resultados: A maioria dos estudos selecionados apresentaram mudanças nos padrões de utilização de substâncias psicoativas. Só no Brasil houve um aumento de 13,1\% no consumo de bebidas alcoólicas, já o número de cigarros diários consumidos por tabagistas aumentou aproximadamente $34 \%$, sem contabilizar outras formas de consumo de tabaco e seus derivados. O consumo de benzodiazepínicos subiu 12,7\% no último ano e houve um crescimento de quase $14 \%$ na comercialização de antidepressivos e estabilizadores de humor. Conclusão: Em análise, foi possível estabelecer uma relação entre ou aumento do consumo de substâncias psicotrópicas e o impacto à saúde mental gerado pela pandemia da Covid-19, que é devido em grande parte ao isolamento social, as grandes mudanças na rotina e no perfil econômico de grande parte da população o que afetou negativamente a saúde mental, um dos pilares da saúde humana derrubado nesse período gerando grande alteração no consumo de medicamentos psicotrópicos, álcool e tabaco. Portanto, atualmente temos um grande problema de saúde pública que pode perdurar durante muito tempo e levar a outros problemas de saúde, sendo assim, são necessárias intervenções dos órgãos governamentais de orientação e promoção à saúde. O presente artigo demonstra a necessidade de novos estudos e relatos capazes de elucidar de maneira consistente 0 rumo dessa problemática, entretanto, consegue exercer um auxílio significativo nas ações de combate à situação.

Palavras-chave: Psicoativos; Covid-19; Saúde Mental.

\section{ABSTRACT}

Objectives: The aim of this study was to conduct a behavioral analysis of the use of licit psychoactive drugs during the Covid-19 pandemic. Methodology: A literature search was conducted of materials available in electronic media from several renowned databases: Elsevier, Google Scholar, Latin American and Caribbean Literature on Health Sciences - LILACS, National Library of Medicine of the United States National Institute of Health (PubMed Central - PMC) and SciELO. A total of 39 materials were 
selected, considering the impact factor of the publications. Results: The selected studies presented changes in the patterns of psychoactive substance use. In Brazil there was an increase of $13.1 \%$ in the consumption of alcoholic beverages, whereas the number of daily cigarettes consumed by smokers increased by approximately $34 \%$, without accounting for other forms of tobacco consumption and its derivatives. Consumption of benzodiazepines rose $12.7 \%$ in the last year and there was a growth of almost $14 \%$ in the marketing of antidepressants and mood stabilizers. Conclusion: In analysis, it was possible to establish a relationship between or increase in the consumption of psychotropic substances and the impact on mental health generated by the Covid-19 pandemic, which is largely due to social isolation, major changes in routine and economic profile of much of the population what negatively affected mental health, one of the pillars of human health overthrown in this period generating great change in the consumption of psychotropic medications, alcohol and tobacco. Therefore, we currently have a major public health problem that can last for a long time and lead to other problems, so interventions are needed from government agencies of guidance and health promotion. This article demonstrates the need for new studies and reports able to consistently elucidate the direction of this problem, however, can exercise significant assistance in actions to combat the situation.

Keywords: Psychoactive Drugs; Covid-19; Mental Health.

\section{INTRODUÇÃO}

O ano de 2020 foi marcado por mudanças que abalaram pilares importantes da sociedade a nível mundial. Supostos casos de pneumonia foram reportados da cidade de Wuhan na China para a Organização Mundial de Saúde (OMS) e, posteriormente, no dia 11 de fevereiro de 2020 identificados como doença Coronavírus 2019 (Covid-19). Estudos realizados, mostraram que 79\% do material genético do "novo vírus" é igual ao da SARS (Síndrome Respiratória Aguda Grave) - vírus que gerou um surto da doença em 2003. Então, no dia 11 de março de 2020, a OMS declarou pandemia de Covid-19 [1]. Após o anúncio, foi gerada uma tensão que modificaria hábitos e comportamentos na rotina das pessoas.

Nessa perspectiva, a imprevisibilidade para a normalidade pós-pandemia em associação ao isolamento social, levou a vulnerabilidade das pessoas a transtornos mentais, no que tange a saúde mental. Segundo Salvetti e Pimenta (2007) [2], diante de uma situação de crise, a fragilidade emocional da população pode levar a respostas que irão interferir de forma negativa, conduzindo a maneiras inadequadas na tentativa de lidar com esse desequilíbrio, como fumar, beber e utilizar medicamentos. Assim, reveste-se de particular importância a manutenção da saúde mental neste período de angústia causado pelo isolamento social advindo da pandemia.

O mais preocupante, contudo, é constatar que há uma alteração significativa no padrão de consumo de psicoativos. Não é exagero afirmar que até mesmo o álcool presente na cerveja, por exemplo, não possui limite seguro para consumo e, de acordo com a OMS (2020) [3], o dano à saúde é proporcional à quantidade ingerida. Então, preocupa o fato de que beber algumas latas de cerveja no fim da noite para alívio do estresse, ocasionado pelo isolamento, possa evoluir para uma dependência de bebida alcoólica e, por conseguinte, afetar a saúde e o bem-estar de milhares de pessoas.

Convém ressaltar que antes mesmo do surto de Covid-19, o consumo excessivo de medicamentos psicotrópicos já era um problema âmbito mundial. A OMS (2017) [4] exemplifica, com informações em escala mundial que mostram o número de casos de pessoas com depressão vem aumentando cerca de $18,4 \%$ nos últimos 10 anos. Revelando então, uma tendência ao aumento no uso dessas substâncias e possivelmente agravada pela pandemia.

Portanto, vê-se a necessidade de um estudo para investigar e analisar a combinação da influência da pandemia causada pelo SARS-CoV-2 na vida das pessoas e o impacto mundial no consumo de psicoativos lícitos.

\section{METODOLOGIA}

Este é um estudo de revisão de literatura integrativa, que versa acerca da utilização de substâncias psicoativas lícitas durante a pandemia da Covid-19 [5]. Desse modo, foram levantados dados através das bases de dados Elsevier, Google Scholar, Literatura Latino Americana e do Caribe em Ciências da Saúde - LILACS, Biblioteca Nacional de Medicina do Instituto Nacional de Saúde dos Estados Unidos (PubMed Central - PMC) e SciELO.

A busca por material foi realizada a partir dos seguintes descritores: psicoativos, psicotrópicos, pandemia da Covid-19, SARS-CoV-2, saúde mental, tabaco e álcool.

O critério de inclusão alinhado para esta pesquisa foi à presença no conteúdo dos textos relacionados às substâncias psicoativas em associação ao consumo durante o período de pandemia, saúde mental durante a quarentena e os desdobramentos do isolamento social. Foram buscados textos escritos em inglês e na ausência desse conteúdo, as palavras chaves foram buscadas em português, entre sites e artigos foram analisados 39 materiais. Consolidou-se os resultados obtidos, reunindo as principais ideias e fatos do assunto publicado, buscando estabelecer relações. Por fim, utilizou-se o fluxo na Figura 2 para estabelecer os critérios de seleção dos materiais utilizados neste estudo.

A composição do trabalho resultou de seleções de artigos, teses, dissertações e publicações on-line no período indicado na Figura 1: 


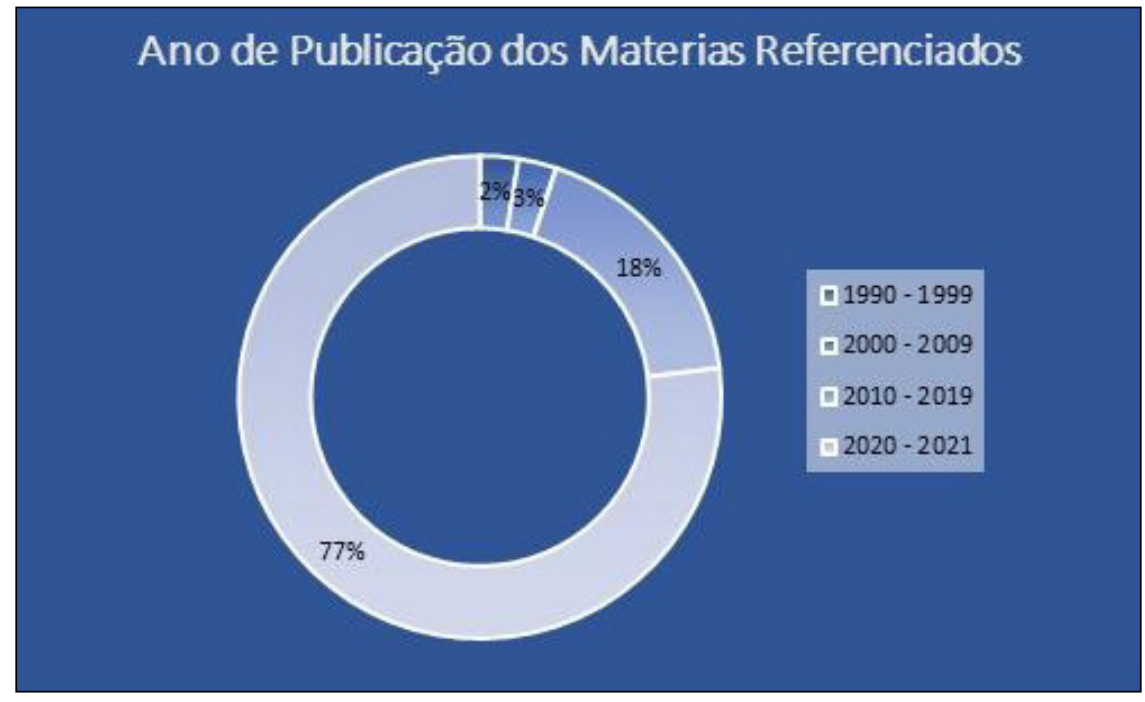

Figura 1: Gráfico ilustrando os anos de publicação dos materiais referenciados.

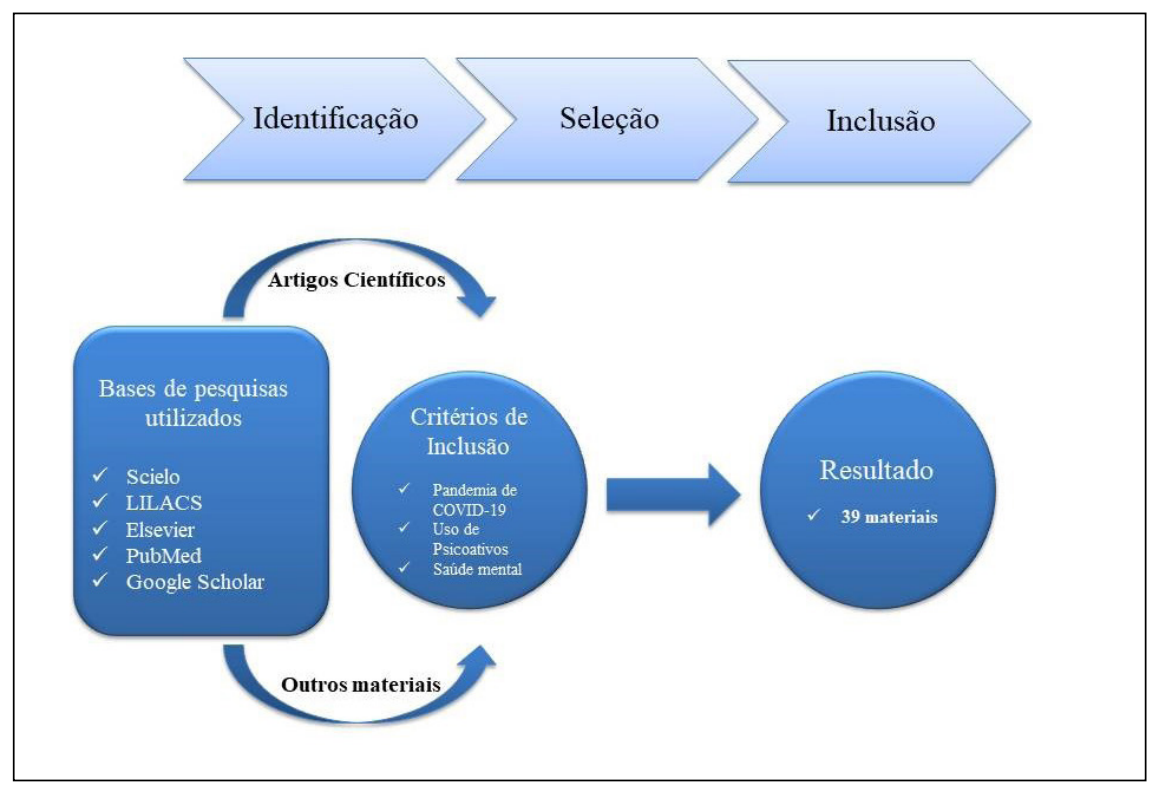

Figura 2: Processo de seleção dos materiais de pesquisa.

\section{Saúde mental durante a pandemia de Covid-19}

Apesar do século 21 ter vivenciado outras pandemias, os eventos desastrosos causados pelo SARS-CoV-2 são inéditos. Diante disso, BBC (2020) [6] relembra que em abril de 2019, um subtipo do vírus influenza disseminou-se para vários países, fragilizando a saúde de milhares de pessoas. Esperava-se, portanto, que quando iniciou a pandemia atual o mundo já estivesse preparado para lidar com este tipo de crise, porém a situação tomou proporções muito maiores.

Sob essa ótica, para controle da crise instaurada com a pandemia, uma das principais medidas de controle e prevenção da doença, causada pelo coronavírus, foi a prática do distanciamento social. Segundo Wilder-Smith et al. (2020) [7] a técnica de isolamento é a separação de pessoas doentes e não-doentes visando reduzir a transmissibilidade da doença. Entretanto, a mudança na rotina e o impedimento de socializar, aliados aos anseios de uma quarentena sem previsão de término, pode levar a caminhos preocupantes no que se diz respeito à saúde mental.

Além do isolamento social ter trazido mudanças nos padrões de comportamento da população, trouxe também consequências econômicas e experiências que levaram a situações elevadas de estresse em muitas áreas, mas principalmente relacionadas a diminuição de recursos na área da saúde para cuidados 
de doenças previamente existentes e acompanhamento dos infectados pela Covid-19. Estudos realizados em condições pandêmicas que relacionam a situação da SARS-CoV-2 e a da SARS, validam que a quarentena contribui para o aumento de transtornos psíquicos [8].

Vale salientar que a ansiedade, quando patológica, pode ser definida como uma resposta emocional que pode levar a um desequilíbrio em questões físicas, mentais e até mesmo sociais. $\mathrm{O}$ estado de ansiedade passa a ser preocupante, quando exagerado e desproporcional ao estímulo, intervindo na qualidade de vida, desempenho das rotinas diárias e a sensação de segurança emocional [9]. O que é percebido, infelizmente, é que a pandemia pode ter exposto a população, e mais especificamente os grupos mais afetados (idosos, doentes crônicos, populações negligenciadas, etc.), a vivenciar a ansiedade em sua forma patológica.

No Brasil, por exemplo, o Ministério da Saúde (2020) [10] divulgou um estudo referente à ansiedade durante a pandemia e foi constatada uma presença elevada de ansiedade $(86,5 \%)$ durante este período. Além disso, outros transtornos foram incluídos na pesquisa, como por exemplo, depressão (16\%).

Pesquisas conduzidas na China ilustram os impactos provocados pela doença, no início do surto, na saúde mental da população em escala nacional. Os dados demonstram que grande parte dos grupos estudados apresentaram problemas psicológicos, como depressão e sintomas severos causados pela ansiedade [8]. Dado a importância a estes dados, é compreendido que havia, desde o início, uma tendência ao aumento de distúrbios mentais juntamente a crise vivenciada pela população mundial. Entretanto, a população a fim de enfrentar esses estressores, podem ser levadas a diferentes maneiras para lidar com a situação, incluindo uso de psicoativos de forma abusiva.

Vale ressaltar que o consumo de psicoativos está intimamente relacionado à predisposição e a situações que vulnerabilizam um indivíduo. Exemplificado essa problemática, Dalpiaz e colaboradores (2014) [11] reportam em sua pesquisa que sintomas como os de depressão, podem incitar indivíduos ao uso de álcool e outras drogas. Ademais, cabe destacar que o uso dessas substâncias contribui para a piora de problemas mentais. Comprovando essa afirmação, Cordeiro e Diehl (2011) [12] relatam que transtornos de humor, ansiedade e depressão são doenças comuns a usuários de drogas.

Em tese, a pandemia da Covid-19 mostrou que diminuir as repercussões psicológicas na população, principalmente nos indivíduos com vulnerabilidades específicas, pode ser um impedimento para que o país lide com o cenário caótico provocado pelo vírus. O artigo de Cullen e colaboradores (2020) [13], retrata a situação relatada anteriormente, afirmando que a saúde mental representa um fator importante na adesão de medidas de saúde pública, como a vacinação, além de ser influente em como as pessoas lidam com a infecção pelo vírus e até mesmo a perda de entes queridos. Nessa perspectiva, é essencial responsabilizar setores específicos para que haja um maior cuidado e preparo para orientação da sociedade como um todo.
No contexto de pensar em planos para redução nos danos à saúde mental, é válido questionar se as autoridades de saúde pública, durante todo o período de pandemia, envolveram esforços que vão além dos danos físicos e biológicos, mas que considerem também, os danos psicológicos. Na China, por exemplo, foram implantadas diretrizes específicas para atenção psicológica a grupos de pessoas com características vulnerabilizadas pelo risco advindo da pandemia [14]. Além disso, deve-se haver uma atenção especial aos pacientes infectados que passaram pelo isolamento, aos familiares que conviveram com o receio de adoecerem ou perder seu ente querido. No Brasil, profissionais da saúde se voluntariaram para atendimentos presenciais e a distância, com o objetivo de impedir desdobramentos futuros que podem levar as pessoas a uso de drogas ou até mesmo danos graves à saúde mental [15].

\section{Consumo de Álcool}

O consumo de álcool, de forma exacerbada, é um problema pertinente atualmente na sociedade. Segundo a Organização PanAmericana da Saúde (OPAS) (2021) [16], o consumo de álcool pode causar uma sobrecarga social, econômica e interferir na saúde do indivíduo, provocando distúrbios mentais, intensificando a ansiedade e depressão, e os distúrbios comportamentais que tem como principais consequências a causa de acidentes de trânsito e violências domésticas associadas à embriaguez. Ademais, seu consumo exacerbado causa dependência e resultam em doenças como, cirrose hepática, doenças cardiovasculares, entre outras disfunções.

Além dos danos citados anteriormente, o uso excessivo deste psicoativo pode prejudicar o sistema imunológico, dificultando o combate de algumas doenças infecciosas, como a síndrome de desconforto agudo, uma das complicações da Covid-19 [17]. Visto isso, a OMS (2020) [3] orienta que a população evite totalmente o uso desta substância para que não haja danos ao sistema imunológico. Reforça também o fato de o consumo de bebidas alcóolicas não eliminar o SARS-CoV-2, e sim aumentar os riscos de contrair o vírus.

Algumas das principais medidas implantadas pelo governo durante a pandemia para controle da transmissão desenfreada da doença, incluem o distanciamento social e o fechamento de serviços não essenciais, como bares, restaurantes, festivais e, em alguns países, lojas especializadas em vendas de bebidas alcoólicas [18]. Tendo isso em vista, é esperado que o consumo de bebidas alcoólicas tenha passado, majoritariamente, de locais públicos para as próprias residências [19]. Esse fator pode ser crítico, considerando que associado aos problemas advindos da epidemia, como problemas financeiros, medo da infecção pelo vírus e o isolamento por si só, faz com que muitas pessoas percam o controle na utilização das bebidas alcoólicas. Em análise dessa problemática, estudos comprovam que exposição a eventos traumáticos levam as pessoas a utilizar o álcool como forma de alívio momentâneo [20].

De acordo com os dados de São Paulo (2021) [21], o 
consumo de bebidas alcóolicas durante a pandemia da Covid-19 aumentou, e só no Brasil houve um aumento de $13,1 \%$, sendo que a média mundial é de $13,5 \%$, obtendo chances de maior frequência no uso da substância em razão dos eventos graves de ansiedade que aumentaram em $73 \%$. Nesse contexto, outros países também demonstraram alterações nos hábitos de consumo de álcool. Durante o período de lockdown, pesquisas mostram o aumento de $31 \%$ na venda de bebidas alcoólicas no Reino Unido e no mesmo artigo, é relatado que um quinto das pessoas que já bebiam antes da quarentena passaram a beber mais diariamente [20]. Na Alemanha, também realizaram uma pesquisa e dentre aproximadamente 2000 integrantes, 35\% ingeriram mais álcool durante $\mathrm{o}$ isolamento social [22].

Frente ao novo contexto social forçadamente introduzido na vida da sociedade, se mostra necessária a intervenção de órgãos públicos e privados em associação aos principais meios de comunicação, para fornecer fontes de informação e orientação para as pessoas. Entretanto, locais com dificuldade de acesso aos meios de comunicação, como a televisão e internet, ainda carecem de uma assistência mais especializada quanto ao assunto do consumo de psicoativos, os marginalizando e fragilizando a lidar sozinhos com as sequelas após este período.

\section{Consumo de Tabaco}

Não é novidade que o consumo de tabaco é uma epidemia de luta constante em todo o mundo. Atualmente, o tabagismo é a principal causa de morte evitável no mundo, além de ser um fator de risco para doenças e infecções respiratórias graves. Muitos países implementaram práticas de controle do tabaco preconizadas pela OMS e, segundo o último relatório, o Brasil já alcançou o mais alto nível no cumprimento dessas estratégias [23]. Entretanto, a pandemia da Covid-19 pode ser vista como um obstáculo neste embate contra o tabaco, visto que a crise impactou aspectos importantes na vida das pessoas.

Com um cenário caótico instaurado pela constante ameaça de infecção pelo vírus SARS-CoV-2, a população tende a buscar formas de se sentir melhor e mais relaxada. O Ministério da Saúde (2019) [24] afirma que a sensação atribuída ao uso do cigarro é a capacidade de oferecer ao fumante um sentimento de conforto momentâneo, além de um escape para lidar com situações desagradáveis. Sendo assim, pressupõe-se que fatores como ansiedade, depressão e a tensão gerada no período de quarentena podem ter influenciado no uso nocivo de tabaco.

É importante comentar que o tabagismo durante a pandemia de uma doença respiratória, é um fator atenuante a ser considerado pelos órgãos globais de saúde, tendo em consideração que as pessoas que fumam passam a ser grupo de risco. Estudos demonstram que a não susceptibilidade a tabaco e fumaças no geral, pode ser um fator prejudicial e agravante em relação a infecção por SARS-CoV-2 [25]. Portanto, além dos efeitos previamente conhecidos, a exposição ao cigarro em meio ao período atual deve ser evitada.

Em análise prática da relação entre tabaco e a infecção pelo vírus, um estudo realizado na China confirmou que fumar está associado com a progressão da doença causada pelo SARS-CoV-2 [26]. Em contrapartida, há dados que demonstram que fumar está associado a baixa susceptibilidade à infecção por SARS-CoV-2, entretanto, não é um fator de proteção ao vírus [27]. Apesar de haver divergências entre as pesquisas, ambos asseguram que fumar não deve ser considerado como uma medida de proteção à saúde. Sendo assim, é de grande importância a investigação comportamental dos fumantes durante os dois últimos anos.

Exemplificando essa problemática em relação ao tabaco no período de lockdown, nos Estados Unidos, houve um acréscimo na venda desses produtos [28]. Na França, um estudo divulgou que mais de um quarto dos fumantes relataram aumento no consumo dessa substância [29]. Não diferentemente de outros países, no Brasil, uma pesquisa realizada pela Fiocruz, evidenciou que $34 \%$ dos fumantes aumentaram o número de cigarros fumados diariamente [30]. Todavia, alguns estudos indicam que a pandemia funcionou como uma forma de motivação para cessar o hábito de fumar [31].

Em suma, o comportamento do uso de tabaco durante a pandemia da Covid-19 foi uma variante cultural, visto que muitos países levaram em consideração que a saúde mental e, consequentemente, o uso descompensado de psicoativos estariam em risco precavendo-se, fechando estabelecimentos que vendem esses produtos, por exemplo. Aliando-se a isso, outra reflexão importante, é a relação de risco entre o tabagismo e a infecção pelo vírus SARS-CoV-2, na qual é preocupante devido a iminente piora dos quadros, entretanto é um medo que levou muitas pessoas a abdicarem do vício estimulando a uma vida mais saudável. Por fim, a luta contra o tabagismo, mesmo após a vacina continua sendo um problema, e os órgãos governamentais juntamente dos meios de comunicação devem investir ainda mais em informação para a população, além de meios assistenciais para os fumantes, como os grupos de apoio.

\section{Consumo de psicofármacos}

O uso nocivo de medicamentos psicotrópicos é uma grave questão de saúde pública. Há registros de organizações como a OMS e o International Narcotics Control Board (INCB), que relacionam o crescimento na utilização desses medicamentos com o aumento nos diagnósticos de transtornos psiquiátricos na população, à introdução de novas substâncias no mercado farmacêutico e às novas indicações terapêuticas de psicofármacos já existentes [32]. No Brasil, por exemplo, em média, são consumidas aproximadamente 500 milhões de apresentações de psicofármacos por ano, com até $70 \%$ podendo representar agentes benzodiazepínicos. Nesse âmbito, sintomas como angústia, insônia, falta de paciência e ansiedade ampliam, como resquício do isolamento social, começando a mudar o fluxo nos consultórios de psicólogos e psiquiatras e, por consequência, afetando o nível de consumo de substâncias psicotrópicas [33]. Nos últimos dois anos, pode-se admitir a magnitude da pandemia e suas consequências como aspectos 
detratores para o aumento da medicalização correlata ao uso de psicofármacos, seja prudente ou não. No Brasil, por exemplo, houve alta nas vendas desses medicamentos: antidepressivos bupropiona (137\%), amitriptilina (41,5\%), escitalopram (37,9\%) e trazodona $(17,4 \%)$, do benzodiazepínico bromazepam (120\%) e do hipnótico zopiclona $(29,3 \%)$ [34]. Especificamente em relação a benzodiazepínicos, São Paulo (2021) [21] aponta um aumento de $12,7 \%$ no consumo dessa classe terapêutica. Tal como, no Canadá, um estudo que analisou a dispensação de medicamentos psicotrópicos, evidenciou um maior número de prescrição de janeiro até setembro de 2020 dessas drogas durante o período de quarentena [35]. Esses fatores corroboram para que a mudança seja desproporcional em relação às tendências esperadas, diferenciando-se das alterações observadas na prescrição de outros medicamentos durante $o$ ano.

Uma pesquisa realizada pela IQVIA comparou um período de janeiro a julho dos anos 2019 e 2020. Notou-se um aumento de quase $14 \%$ na comercialização de antidepressivos e estabilizadores de humor, utilizados em indivíduos com depressão e distúrbio bipolar. Neste mesmo estudo, foi identificada uma elevada proporção quanto às vendas, no qual em 2019 foram 56,3 milhões, em 2020 foi de 65,1 milhões [36].

Atividades de telefarmácia foram inseridas no contexto da pandemia da Covid-19 com a finalidade de promover o uso racional de medicamentos, visto que há o auxílio do profissional farmacêutico e médico via telefone ou e-mail, instruções para acesso e até mesmo a fiscalização da dispensação dessas substâncias [37]. Diante desses dados, essas orientações são de importância aos usuários de psicotrópicos, em vista que em meio a esta epidemia, encontram-se debilitados mentalmente e fisicamente e necessitam de atenção farmacêutica, que assegura uma assistência integral, possibilitando a promoção e recuperação da saúde com o uso de substâncias de forma adequada. A International Union of Basic and Clinical Pharmacology (IUPHAR) (2021) [38], fundou, no dia 6 de maio de 2021, o World Smart Medication Day (Dia Mundial da Medicação Inteligente), com o propósito de orientar os indivíduos sobre um uso mais racional, eficaz e seguro dos medicamentos, expandindo a visão sobre o tema.

\section{DISCUSSÃO E RESULTADOS}

Dianteaos dados apresentadosé possível consideraroaumento da utilização do tabaco, do álcool e dos psicofármacos, devido à epidemia da Covid-19. Os antidepressivos e estabilizadores de humor, conforme CFF (2020) [36], por exemplo, tiveram um crescimento em sua venda de quase $14 \%$, que se dá devido à instabilidade emocional durante o lockdown. Considerando esta informação, observa-se uma maior proporção nos casos de ansiedade de $86,5 \%$, depressão $16 \%$ e estresse pós-traumático $45,5 \%$, de forma que os indivíduos sintam satisfação e bem-estar momentâneo durante a ingestão desses medicamentos, como o uso dos benzodiazepínicos, o mais utilizado [39].

Da mesma maneira, identifica-se um crescimento na venda e seguidamente, na ingestão de álcool em 13,1\% no Brasil, e 13,5\% como média mundial, isso desencadeado por consequência da crise financeira com a queda da renda e o desemprego, medo e o isolamento social, promovendo o descontrole, e aumentando as ocorrências de distúrbios comportamentais, como violência doméstica que foi muito noticiada durante o período de isolamento $[3,21]$.

Com o tabaco não foi diferente, houve um crescimento nas vendas o que é devido ao aumento no consumo diário pelos usuários desta droga, como forma de escape, por consequência relaxamento momentâneo e alívio em tempo deste lapso [30].

Desse modo, percebe-se que a pandemia de Covid-19 surpreendeu negativamente a vida de bilhões de pessoas no mundo todo, impactando principalmente questões relacionadas à saúde. Nesse sentido, o emocional e o psíquico são fatores que foram profundamente abalados nesse período, levando ao consumo desses psicoativos [2].

\section{CONCLUSÃO}

A pandemia da Covid-19 está além da globalização de um vírus que se propaga de maneira descontrolada e em velocidade nunca vista anteriormente. A pandemia expõe mazelas da sociedade na mesma amplitude que sua propagação. Salientamos aqui, que além da pandemia de um vírus, temos muitas outras pandemias em curso na mesma rapidez ou até maior, pois já existiam antes e foram apenas fortalecidas durante esse período. Atualmente temos a pandemia da fome, da vulnerabilidade e desigualdade social, da educação e o impacto negativo sobre ela expondo ainda mais a falta de equidade na educação principalmente em países subdesenvolvidos e a saúde mental da sociedade que está sendo afetada por múltiplos fatores.

Outro grande problema de nível pandêmico que temos é o uso abusivo de substâncias psicoativas lícitas como o álcool, tabaco e também de medicamentos psicotrópicos que são consumidos e prescritos de forma irracional. A combinação entre a pandemia da Covid-19 e o uso dessas substâncias psicoativas se tornaram uma preocupação a nível mundial, dado que essa parte da população faz parte do grupo vulnerável é a que está mais suscetível a diversos riscos neste período, como o uso indevido e a automedicação. Sendo assim, a análise sugere que o impacto social da pandemia — que resulta na piora da desigualdade, de problemas financeiros e das condições de saúde mental, sobretudo entre populações já fragilizadas - representa o aumento no consumo de drogas e deve-se investir para além desse período em promoções à saúde para o uso racional de medicamento e campanhas para a redução de tabaco e álcool.

\section{REFERÊNCIAS}

1. Anand KB, Karade S, Sen S, Gupta RM. SARS-CoV-2: Camazotz's Curse. MJAFI. 2020;76(2):136-41.

2. Salvetti MD, Pimenta CA. Dor crônica e a crença de autoeficácia. REEUSP. 2007;41(1):135-40. 
3. World Health Organization. World Health Organization [Internet]. Alcohol and COVID: what do you need to know?; 2020. Disponível em: https://www.euro.who.int/_data/assets/ pdf_file/0010/437608/Alcohol-and-COVID-19-what-you-need-toknow.pdf

4. World Health Organization. World Health Organization [Internet]. Depression and other common mental disorders: global health estimates; 2017. Disponivel em: https://apps.who. int/iris/handle/10665/254610

5. Souza MT, Silva MD, Carvalho RD. Integrative review: what is it? How to do it? Einstein (São Paulo). 2010;8(1):102-6.

6. Barifouse R. BBC News Brasil [Internet]. Por que o H1N1 não parou economias como a pandemia de coronavírus? BBC News Brasil; 2020. Disponível em: https://www.bbc.com/ portuguese/internacional-52078906

7. Wilder-Smith A, Freedman DO. Isolation, quarantine, social distancing and community containment: pivotal role for old-style public health measures in the novel coronavirus (2019-nCoV) outbreak. JTM. 2020;27(2).

8. Wang C, Pan R, Wan X, Tan Y, Xu L, Ho CS, Ho RC. Immediate Psychological Responses and Associated Factors during the Initial Stage of the 2019 Coronavirus Disease (COVID-19) Epidemic among the General Population in China IJERPH. 2020;17(5):1729.

9. Allen AJ, Leonard H, Swedo SE. Current Knowledge of Medications for the Treatment of Childhood Anxiety Disorders. J. Am. Acad. Child Adolesc. Psychiatry. 1995;34(8):976-86.

10. Ministério da Saúde. Ministério da Saúde [Internet]. Plano de contingência nacional para infecção humana pelo novo Coronavírus 2019-nCoV: centro de operações de emergências em saúde pública (COE-nCoV); 2020. Disponível em:http:// portalarquivos2.saude.gov.br/images/pdf/2020/fevereiro/07/planocontingencia-coronavirus-prelo.pdf.

11. Dalpiaz AK, Jacob MHVM, Silva KD, Bolson MP, Hirdes Alice. Fatores associados ao uso de drogas: depoimentos de usuários de um CAPS AD. Aletheia. 2014; 45: 56-71.

12. Diehl A, Cordeiro DC, Laranjeira R. Dependência Química: Prevenção, Tratamento e Políticas Públicas. Comorbidades psiquiátricas (pp. 106-118). Brasil, 2011, Artmed Editora.

13. Cullen W, Gulati G, Kelly BD. Mental health in the COVID-19 pandemic. QJM. 2020;113(5):311-2.

14. National Health Commission of China. National Health Commission of China [Internet]. Principles of the emergency psychological crisis interventions for the new coronavirus pneumonia; 2020. Disponível em: http://www.nhc.gov.cn/jkj/ s3577/202001/6adc08b966594253b2b791be5c3b9467

15. Ministério da Saúde. Ministério da Saúde [Internet]. Ministério da Saúde divulga resultados preliminares de pesquisa sobre saúde mental na pandemia; 2020. Disponível em: https://antigo.saude.gov.br/noticias/agencia-saude/47527ministerio-da-saude-divulga-resultados-preliminares-de-pesquisasobre-saude-mental-na-pandemia

16. Organização Pan-Americana da Saúde. Organização Pan-Americana da Saúde [Internet]. Álcool; 2020. Disponível em: https://www.paho.org/pt/node/4825

17. Organização Pan-Americana da Saúde. Organização PanAmericana da Saúde [Internet]. $\mathbf{O}$ consumo de bebidas durante a pandemia; 2020. Disponível em: https://www.paho.org/pt/ noticias/12-11-2020-pesquisa-da-opas-em-33-paises-aponta-quequase-metade-dos-entrevistados-no

18. Pan American Health Organization. Pan American Health Organization [Internet]. Response to the COVID-19 Pandemic in the Americas; 2021. https://iris.paho.org/bitstream/ handle/10665.2/53540/PAHOPHEHEOCOVID-19210008_eng. pdf? sequence $=1$ \&amp;isAllowed $=y$

19. Reynolds J, Wilkinson C. Accessibility of 'essential' alcohol in the time of COVID $\square$ 19: Casting light on the blind spots of licensing? Drug and Alcohol Review. 2020;39(4):305-8.

20. The Lancet Gastroenterology \& Hepatology. Drinking alone: COVID-19, lockdown, and alcohol-related harm. Lancet Gastroenterol Hepatol. 2020;5(7):625.

21. Secretaria Municipal da Saúde. Cidade de São Paulo Saúde [Internet]. Consumo de álcool e drogas na pandemia é tema de evento on-line; 2021. https://www.prefeitura.sp.gov.br/ cidade/secretarias/saude/noticias/?p=314429

22. Koopmann A, Georgiadou E, Kiefer F, Hillemacher T. Did the General Population in Germany Drink More Alcohol during the COVID-19 Pandemic Lockdown? Alcohol. 2020;55(6):698699.

23. World Health Organization. World Health Organization [Internet]. Who report on the global tobacco epidemic, 2021; 2021.: https://apps.who.int/iris/bitstream/hand le/10665/343287/9789240032095-eng.pdf?sequence=1\&amp;

24. Governo do Brasil. Saúde Brasil [Internet]. Seu vício no cigarro pode estar encobrindo o quê? 2019. Disponível em: https://saudebrasil.saude.gov.br/eu-quero-parar-de-fumar/seuvicio-no-cigarro-pode-estar-encobrindo-o-que

25. Silva AL, Moreira JC, Martins SR. COVID-19 e tabagismo: uma relação de risco. CSP. 2020;36(5).

26. Patanavanich R, Glantz SA. Smoking is associated with COVID-19 progression: a meta-analysis. Nicotine Tob Res. 2020;22(9):1653-6.

27. Paleiron N, Mayet A, Marbac V, Perisse A, Barazzutti $H$, Brocq FX, Janvier F, Dautzenberg B, Bylicki O. Impact of Tobacco Smoking on the Risk of COVID-19: A Large-Scale Retrospective Cohort Study. Nicotine Tob Res. 2021;23(8):1398-1404.

28. Yach D. Tobacco Use Patterns in Five Countries During the COVID-19 Lockdown. Nicotine Tob Res. 2020;22(9):16711672.

29. Santé publique France [Internet]. Tabac, Álcool: quell impact du confinement sur la consommation des Français?; 2020 [citado 19 set 2021]. Disponível em: https://www. santepubliquefrance.fr/presse/2020/tabac-alcool-quel-impact-duconfinement-sur-la-consommation-des-francais

30. Mazzi C. O Globo [Internet]. Consumo de cigarro aumentou para $34 \%$ dos fumantes brasileiros durante a pandemia, diz pesquisa da Fiocruz; 2020. Disponível em: https:// oglobo.globo.com/brasil/consumo-de-cigarro-aumentou-para- 
34-dos-fumantes-brasileiros-durante-pandemia-diz-pesquisa-dafiocruz-1-24583015

31. Klemperer, EM, West JC, Peasley-Miklus C, Villanti AC. Change in Tobacco and Electronic Cigarette Use and Motivation to Quit in Response to COVID-19. Nicotine Tob Res. 2020;22(9):1662-1663.

32. Wanderley TC, Cavalcanti AL, Santos S. Práticas de saúde na atenção primária e uso de psicotrópicos: uma revisão sistemática da literatura. cmbio. 2013;12(1):121-6.

33. Laureano FRC; Amaral WN; Amaral Filho WN; Falone VE. Medicamentos Psicotrópicos: Uso, Prescrição e Controle. Rev. goiana med., 2015; 47, 22-26.

34. Alves AM, Couto SB, Santana MP, Baggio MRV, Gazarini L. Medicalização do luto: limites e perspectivas no manejo do sofrimento durante a pandemia. CSP. 2021; 37(9): e00133221.

35. Stall NM, Zipursky JS, Rangrej J, Jones A, Costa AP, Hillmer MP, Brown K. Assessment of Psychotropic Drug Prescribing Among Nursing Home Residents in Ontario, Canada, During the COVID-19 Pandemic. JAMA Intern Med. 2021;181(6):861863.

36. Conselho Federal de Farmácia. Conselho Federal de Farmácia [Internet]. Venda de medicamentos psiquiátricos cresce na pandemia; 2020. https://www.cff.org.br/noticia. php?id $=6015 \& a m p ;$ titulo $=$ Venda + de + medicamentos + psiquiátricos+cresce+na+pandemia

37. Lula-Barros, DS; Damascena HL. Assistência farmacêutica na pandemia da Covid-19: uma pesquisa documental. TES. 2021; 19, e00323155.

38. International Union of Basic \& Clinical Pharmacology. International Union of Basic \& Clinical Pharmacology [Internet]. World smart medication day; 2021. Disponível em: https:// iuphar.org/clinical-division/world\%20smart-medication-day

39. Conselho Federal de Farmácia. Conselho Federal de Farmácia [Internet]. Uso racional de psicotrópicos durante a pandemia. 2020. Disponível em: https://www.cff.org.br/noticia. php?id=5951 